\title{
Diagnostic Performance and Inter-Observer Agreement of 4-Dimensional Computed Tomography Parathyroid Scans in Patients with Primary and Secondary Hyperparathyroidism
}

\author{
Bundhit Tantiwongkosi',2, Frank R. Miller², Viet D. Nguyen1, Kathleen E. Hands 3 , \\ Boyce B. Oliver ${ }^{4}$, Alfredo A. Santillan ${ }^{5}$, Wilson B. Altmeyer ${ }^{1}$, Achint K. Singh ${ }^{1}$, Fang Yu ${ }^{6}$ \\ ${ }^{1}$ Radiology Department, University of Texas Health San Antonio, San Antonio, TX, USA \\ ${ }^{2}$ Otolaryngology, Head Neck Surgery Department, University of Texas Health San Antonio, San Antonio, TX, USA \\ ${ }^{3}$ Thyroid and Endocrine Center of South Texas, San Antonio, TX, USA \\ ${ }^{4}$ Surgical Oncology Department, University of Texas Health San Antonio, San Antonio, TX, USA \\ ${ }^{5}$ Surgical Oncology Department, Texas Oncology, San Antonio, TX, USA \\ ${ }^{6}$ Radiology Department, University of Texas Southwestern, Dallas, TX, USA \\ Email: tantiwongkos@uthscsa.edu
}

How to cite this paper: Tantiwongkosi, B., Miller, F.R., Nguyen, V.D., Hands, K.E., Oliver, B.B., Santillan, A.A., Altmeyer, W.B., Singh, A.K. and Yu, F. (2019) Diagnostic Performance and Inter-Observer Agreement of 4-Dimensional Computed Tomography Parathyroid Scans in Patients with Primary and Secondary Hyperparathyroidism. Open Journal of Radiology, 9, 82-91. https://doi.org/10.4236/ojrad.2019.91008

Received: February 16, 2019

Accepted: March 10, 2019

Published: March 13, 2019

Copyright $\odot 2019$ by author(s) and Scientific Research Publishing Inc. This work is licensed under the Creative Commons Attribution International License (CC BY 4.0).

http://creativecommons.org/licenses/by/4.0/

Open Access

\begin{abstract}
Background: 4D-CT has been used to localize the parathyroid adenomas and hyperplasia since 2006 as a second line study after TC-99 m MIBI and ultrasonography. However, multiple studies have shown that $4 \mathrm{D}-\mathrm{CT}$ is a robust imaging method with high diagnostic accuracy, becoming increasingly popular among surgeons and radiologists. Purpose: To assess the diagnostic performance of $4 \mathrm{D}-\mathrm{CT}$ scans to identify the pathologic gland(s), using pathology and intraoperative findings as gold standards. Methods. We analyzed patients with primary and secondary hyperparathyroidism who had intraoperative reports, pathology, parathyroid hormone levels, and preoperative 4D-CT. Histology, surgical findings, and decreased parathyroid hormone levels were used as gold standards. The sensitivity, specificity, positive predictive value (PPV), negative predictive value (NPV), accuracy and 95\% confidence interval were calculated. Fleiss' kappa was used to assess the inter-observer agreement. Results: Sixty-seven patients were included. Sixty-two patients had a single adenoma, and five patients had a multiple gland disease (adenomas or hyperplasia). A total of 72 glands were proven to have parathyroid adenomas or hyperplasia. The sensitivity, specificity, PPV, NPV and accuracy are 85\%, $97 \%, 96 \%, 87 \%$ and $91 \%$ for lateralization and $76 \%, 96 \%, 85 \%, 92 \%$ and $90 \%$
\end{abstract}


for quadrant localization, respectively in single-gland disease. The sensitivity, specificity, PPV, NPV and accuracy are $88 \%, 100 \%, 100 \%, 50 \%$ and $90 \%$ for lateralization and $71 \%, 100 \%, 100 \%, 60 \%$ and $80 \%$ for quadrant localization respectively in multiple-gland disease. Fleiss' kappa value is 5.6 (moderate inter-observer agreement). Conclusion: $4 \mathrm{D}-\mathrm{CT}$ is a robust method in the localization of hyperfunctioning parathyroid glands with high accuracy and at least moderate inter-observer agreement.

\section{Keywords}

Computed Tomography, Parathyroid, 4-Dimensional, Hyperparathyroidism

\section{Introduction}

Primary hyperthyroidism (PHPT) is characterized by unregulated excessive secretion of parathyroid hormone (PTH) resulting in hypercalcemia, most often due to a single parathyroid adenoma (85\%), and less frequently by multiple adenomas/hyperplasia $(10 \%-15 \%)$ and carcinomas $(<1 \%)$ [1]. Secondary hyperparathyroidism (SHPT) occurs when the parathyroid glands are stimulated by chronic hypocalcemia, usually in the setting of chronic renal failure [2]. Parathyroidectomy is the only curative treatment for symptomatic PHPT [3] and medically refractory SHPT [4]. Historically, this was carried out through bilateral neck exploration to identify the hyperfunctioning lesions. Although effective from a therapeutic standpoint, this technique is associated with higher rates of complications compared to focused surgical approaches [5].

In an effort to decrease the rates of complications as well as the length of hospital stays and medical costs, less invasive surgical procedures have been developed. Directed parathyroidectomy is one such technique, which is frequently combined with pre-operative and intra-operative localization of parathyroid lesions and intra-operative parathyroid hormone (PTH) levels [6]. A serial decrease in PTH levels over 50\% and eucalcemia at six months after parathyroidectomy are diagnostic for cure [7]. Imaging modalities traditionally used for preoperative localization include technetium-99 m labeled sestamibi-SPECT (MIBI) and ultrasound (US). Although both modalities exhibit good specificity, they have relatively low sensitivity for lesion detection especially in multigland disease [8]. Ultrasound is further limited in its ability to assess mediastinal lesions and is highly operator-dependent, while sestamibi-SPECT is limited by spatial resolution and variabilities in imaging techniques [9].

Interest in new imaging techniques that offer increased anatomic coverage as well as improved spatial resolution has led to the development of $4 \mathrm{D}-\mathrm{CT}$. This dynamic multiphase CT imaging technique is based on the principle of altered attenuation of parathyroid lesions (adenoma(s) and hyperplasia) during noncontrast, arterial and venous phases [10]. In theory, 4D-CT may provide precise localization of lesions allowing for minimally invasive surgery, detect ectopic le- 
sions (such as mediastinal lesions) that may alter the surgical approach, and also enhance the evaluation of nearby structures (such as thyroid glands) for preoperative planning [11]. Multiple previous studies have shown a high accuracy of $4 \mathrm{D}-\mathrm{CT}$ in localizing abnormal glands up to $93.7 \%$ [11] with a sensitivity of $89 \%$ and a positive predictive value up to $93.5 \%$ [8].

Our goal is to evaluate the diagnostic accuracy and inter-observer agreement of $4 \mathrm{D}$ CT parathyroid scan in preoperative localization of parathyroid lesions in patients with primary and secondary hyperparathyroidism.

\section{Materials and Methods}

\subsection{Patients}

This Institutional Review Board-approved retrospective study was performed with a waiver of informed consents in accordance with the Health Insurance Portability and Accountability Act. Inclusion criteria were patients with biochemically confirmed primary and secondary hyperparathyroidism without previous neck surgeries, had preoperative $4 \mathrm{D}$-CT parathyroid scans performed between June 2011 to September 2017, parathyroidectomy, and pathologically confirmed parathyroid adenoma(s) or hyperplasia. A decrease of intra-operative PTH $($ IOPTH $)>50 \%$ from the baseline and eucalcemia at six months after parathyroidectomy confirm cure status [7]. A total of 67 patients met inclusion criteria (50 women, 17 men; mean age, 55 years; age range, 31 - 74 years).

\subsection{D-CT Parathyroid Scan}

Patients were imaged in the supine position with head first, arms down using a 64-slice CT scanner (Philips, Brilliance 128 slice scanner). The scanning parameters were as follows: field of view covered from the mandibular angle to the tracheal bifurcation parallel to the hard palate, $140 \mathrm{kVp}, \mathrm{mA}$ adjusted by automatic exposure control (AEC) with a range of $180-280 \mathrm{~mA}, 1.25 \mathrm{~mm}$ slice thickness (reconstructed into 1-mm), 0.4 second rotation time, 3.1 sec scan time, and a pitch of 1.265. Dynamic three phases included precontrast, immediate or arterial (25 seconds) and venous phases ( 85 seconds) after intravenous administration of $100-120 \mathrm{ml}$ of iodinated contrast material (Omnipaque 300; GE Healthcare Inc., Princeton, NJ), rate $4 \mathrm{ml}$ per second, followed by $25 \mathrm{ml}$ of saline chaser, via 18 gauge needles placed at the antecubital fossa. All phases were performed with breath holding techniques to reduce motion artifacts. Axial, coronal and sagittal 1-mm reformations were generated and sent to PACS for interpretation.

\subsection{Image Interpretation and Analysis}

$4 \mathrm{D}-\mathrm{CT}$ studies were retrospectively reviewed independently by three neuroradiologists (B.T., W.B.A., and A.C. with 7, 5 and 4 years of neuroradiology experience; B.T. and W.B.A. have Certificates of Added Qualification). The readers were aware that the cases had clinical HPT but were blinded to the final lesion location and pathology results. If there were discrepancies among the readers, 
the final read was determined by consensus. The following data was recorded: presence or absence of parathyroid lesion, location (right, left, upper pole, and lower pole relative to the thyroid glands, or ectopic locations), size, fat plane between the lesions and the thyroid gland, and density (Housefield unit) of the candidate lesions relative to the thyroid gland. Positive findings for parathyroid adenomas/hyperplasia are soft tissue masses that are intrinsically hypodense compared to the thyroid, intense enhancement on arterial phase, and washout on venous phase. Lesions were classified as lymph nodes if they show progressive enhancement, or thyroid nodules if they were isodense to thyroid and adhered to the thyroid glands. If streak artifacts from the intravenous contrast compromised visualization of the lesions, and falsely decreased the density of the lesion in the arterial phase, the lesions were carefully inspected on noncontrast and venous phases.

\subsection{Statistical Analysis}

Performance of $4 \mathrm{D}$-CT was evaluated based on the determination of the presence of the parathyroid lesions, laterality (right or left), and quadrant (right superior, right inferior, left superior and left inferior) with respect to the operative and histological findings.

\subsubsection{Lateralization Analysis}

The performance of $4 \mathrm{D}-\mathrm{CT}$ in the localization of the lesion to the right or left side of the neck was evaluated. If the lesion on $4 \mathrm{D}-\mathrm{CT}$ was found to be ipsilateral to the surgical finding, the $4 \mathrm{D}-\mathrm{CT}$ result was considered as a true positive on that side and true negative on the contralateral side. On the other hand, if the surgical and imaging findings were incongruent, the 4-D CT finding was considered as false positive on that side and false negative on the other. If the lesions are bilateral but the 4-D CT localized the lesion to only one side, the 4-D CT result was considered true positive on that side and false negative on the other. If there was only one lesion but 4-D CT finding was bilateral, the 4-D CT result was considered true positive on the side of the actual lesion and false positive on the other.

\subsubsection{Quadrant Localization Analysis}

The performance of 4-D CT in the localization of the lesion to the level of the quadrant (right upper, right lower, left upper and left lower quadrant) was analyzed in a similar fashion as lateralization.

Sensitivity, specificity, positive predictive value (PPV), negative predictive value (NPV), accuracy and 95\% confidence interval were calculated by utilizing Excel 2010 (Microsoft, Redmond, Wash). The causes of all false negative or false positive results were determined (patient factors, technical factors or lesion factors). Fleiss' kappa was used to assess the inter-observer agreement.

\subsection{Results}

Demographic information of the 67 patients included in the studies is shown in 
Table 1. Sixty-two patients (93\%) had single-gland adenomas. Of the five patients (7\%) with multiple-gland lesions, four had double adenomas, and one had 4-gland hyperplasia. Diagnostic performance of 4D-CT in lesion lateralization and quadrant determination is shown in Table 2. The sensitivity, specificity, PPV, NPV and accuracy are 85\%, 97\%, 96\%, 87\% and 91\% for lateralization and $76 \%, 96 \%, 85 \%, 92 \%$ and $90 \%$ for quadrant localization, respectively in single-gland disease. The sensitivity, specificity, PPV, NPV and accuracy are $88 \%$, $100 \%, 100 \%, 50 \%$ and $90 \%$ for lateralization and $71 \%, 100 \%, 100 \%, 60 \%$ and $80 \%$ for quadrant localization respectively in multiple-gland disease. The three neuroradiologists reached moderate inter-observer agreement with an overall

Table 1. Patient, lesion and laboratory data.

\begin{tabular}{lc}
\hline \multicolumn{1}{c}{ Parameters } & Units \\
\hline Total patients (n) & 67 \\
Female (n) & $50(75 \%)$ \\
Male (n) & $17(25 \%)$ \\
Age (years) & 55 \\
BMI (Kg/m ${ }^{2}$ ) & 31 \\
Lesion weight (mg) & 492 \\
Total glands resected (n) & 76 \\
$\quad$ Single-gland adenoma (n) & $62(93 \%)$ \\
$\quad$ Multiple-gland lesions (n) & $5(7 \%)$ \\
$\quad$ Two-gland ademonas (n) & $3(4 \%)$ \\
$\quad$ Four-gland hyperplasia (n) & $2(3 \%)$ \\
PTH (pg/ml) & \\
Preoperative & 297 \\
Postoperative & 36 \\
\hline
\end{tabular}

Table 2. Diagnostic performance analysis.

\begin{tabular}{|c|c|c|c|c|c|c|c|c|c|}
\hline & $\mathrm{TP}$ & $\mathrm{TN}$ & FP & FN & Sensitivity $(95 \% \mathrm{CI})$ & Specificity (95\% CI) & PPV (95\% CI) & NPV (95\% CI) & Accuracy $(95 \%$ CI) \\
\hline \multicolumn{10}{|l|}{$\begin{array}{c}\text { Single gland (62 } \\
\text { patients, } 62 \text { lesions) }\end{array}$} \\
\hline $\begin{array}{l}\text { Lateralization } \\
\text { (124 sides) }\end{array}$ & 53 & 60 & 2 & 9 & $85 \%(74 \%-93 \%)$ & $97 \%(89 \%-99 \%)$ & $96 \%(87 \%-97 \%)$ & $87 \%(77 \%-94 \%)$ & $91 \%(84 \%-95 \%)$ \\
\hline \multicolumn{10}{|c|}{$\begin{array}{l}\text { Quadrant localization } \\
\text { (248 quadrants) }\end{array}$} \\
\hline & 47 & 178 & 8 & 15 & $76 \%(63 \%-86 \%)$ & $96 \%(92 \%-98 \%)$ & $85 \%(73 \%-94 \%)$ & $92 \%(86 \%-96 \%)$ & $90 \%(86 \%-94 \%)$ \\
\hline \multicolumn{10}{|c|}{$\begin{array}{c}\text { Multiple glands } \\
\text { (5 patients, } 14 \text { lesions) }\end{array}$} \\
\hline $\begin{array}{l}\text { Lateralization } \\
\text { (10 sides })\end{array}$ & 8 & 1 & 0 & 1 & $88 \%(52 \%-99 \%)$ & $100 \%(20 \%-100 \%)$ & $100 \%(70 \%-100 \%)$ & $50 \%(1 \%-90 \%)$ & $90 \%(51 \%-99 \%)$ \\
\hline $\begin{array}{c}\text { Quadrant } \\
\text { (20 quadrants) }\end{array}$ & 10 & 6 & 0 & 4 & $71 \%(42 \%-92 \%)$ & $100 \%(50 \%-100 \%)$ & $100 \%(69 \%-100 \%)$ & $60 \%(26 \%-88 \%)$ & $80 \%(56 \%-94 \%)$ \\
\hline
\end{tabular}

TP true positive, TN true negative, FP false positive, FN false negative, CI confidence interval, PPV positive predictive value, NPV negative predictive value. 
Fleiss' kappa of 0.56. False positive and false negative findings occurred in $17 \mathrm{pa}-$ tients (25.3\%) and 19 lesions (25\%). Multiple factors were responsible for the misinterpretation of the $4 \mathrm{D}-\mathrm{CT}$ imaging. These factors may be classified into lesion, patient, and technical factors, and more than one factor as shown in Table 3. Patient factors include large body habitus ( $29 \%$ of the patients, $26 \%$ of the lesions) and motion artifact (5\% of the patients, $5 \%$ of the lesions). Technical factors include suboptimal bolus injection (18\% of patients, $16 \%$ of lesions) and streak artifact (18\% of patients, $16 \%$ of lesions). Major lesion factors are small lesions ( $24 \%$ of patients, $32 \%$ of lesions) and lack of fat planes between the lesions and the thyroid gland ( $29 \%$ of patients, $37 \%$ of lesions).

\section{Discussion}

Parathyroidectomy is the only curative treatment for PHPT and medically intractable SHPT with a greater than 95\% success rate when performed by experienced surgeons [7]. Historically, bilateral neck exploration (BNE) was performed to remove the culprit hyperfunctioning parathyroid glands [12]. With current intraoperative PTH measurements and preoperative localizing 4D-CT parathyroid scans, surgeons can now perform minimally invasive parathyroidectomy (MIP) focusing on only one quadrant of the neck or even specific locations. Although the success rate of BNE and MIP is equivalent, the operative time, hospital stay, operative pain, and complication rates is significantly less for MIP [13].

Multiple studies have shown that the 4D-CT has high sensitivity (77\% - 92\%; $85 \%$ our study) and accuracy (80\% - 97\%; 91\% our study) in lateralizing single gland lesions [11] [14] [15] [16] [17]. The diagnostic performance is slightly lower for precisely localizing a single lesion to a quadrant, with a sensitivity and accuracy of $57 \%$ - 98\% (76\% our study) and 79\% - 92\% (90\% our study), respectively. Compared to single-gland localization, the diagnostic performance for lateralizing and localizing multiple gland lesions is lower. Chazen and Galvin et al. reported overall sensitivity and accuracy of $43 \%-53 \%$ ( $71 \%-88 \%$ our study) and $89 \%$ ( $80 \%$ - 90\% our study), respectively [14] [18].

Table 3. Factors causing 4D CT misinterpretation.

\begin{tabular}{lcc}
\hline & Patients $(\mathrm{n}=17)$ & Lesions $(\mathrm{n}=19)$ \\
\hline $\begin{array}{l}\text { Patient factors } \\
\text { Large body habitus }\end{array}$ & $5(29 \%)$ & $5(26 \%)$ \\
Motion artifact & $1(5 \%)$ & $1(5 \%)$ \\
Technical factors & & $3(16 \%)$ \\
Suboptimal bolus injection & $3(18 \%)$ & $3(16 \%)$ \\
Streak artifact & $3(18 \%)$ & \\
Lesion factors & & $3(16 \%)$ \\
Multiple lesions & $1(5 \%)$ & $6(32 \%)$ \\
Small lesions & $4(24 \%)$ & $7(37 \%)$ \\
Lack of fat plane & $5(29 \%)$ & $2(11 \%)$ \\
Atypical enhancement & $2(12 \%)$ & $2(11 \%)$ \\
Pudenculated thyroid nodule & $2(12 \%)$ & \\
\end{tabular}


Multiple challenging factors potentially limit the diagnostic performance of 4D-CT which may be classified into three main categories: patient, technique and lesion factors. More than one factor could be responsible for incorrect lesion localization. Large body habitus may result in beam hardening artifact and increased image noise making separation of the lesion from the adjacent normal structures difficult. The lesion would also appear to enhance less than usual. Increasing tube current (mAs) and decreasing rotation speed will decrease image noise at the expense of increased radiation [19]. For patients with thick shoulders, we request the individual to relax and position their shoulders downwards while placing towels between the scapulae as suggested by Hoang et al. [20]. Motion artifact is common in daily practice but contributed to only $5 \%$ of missed lesions in our study. This may be addressed by coaching the patient for breath holding/quiet breathing and abstaining from swallowing during scanning.

Sixteen percent of missed lesions were due to suboptimal opacification during the arterial and venous phases. This may be attributed to incorrect placement of the region of interest, small intravenous access cannula $(22 \mathrm{G})$, and low rate of injection (less than $3 \mathrm{ml} / \mathrm{sec}$ ) which lead to suboptimal enhancement of the lesions. Solutions are to correctly identify the location of the region of interest (aorta) as well as using appropriate cannula size (18-20 G) and appropriate rate of injection ( $5 \mathrm{ml} / \mathrm{sec})$.

Streak artifact from the presence of intravenous iodinated contrast in the subclavian vein, brachiocephalic vein, and superior vena cava during arterial phase also limits visualization of lesions (Figure 1), falsely decreasing the density of arterially enhancing lesions. Two lesions in our study were missed due to such streaking artifact from the intravenous contrast. The saline chasing technique has been proposed to address this pitfall and can be performed by injecting 25 $\mathrm{ml}$ of normal saline after intravenous contrast injection [20] [21]. One lesion was obscured by streak artifact from metallic plates and screws (previous anterior cervical discectomy and fixation). In this scenario, patients with metallic prosthesis in the lower neck may benefit from ultrasound and Tc-99 $\mathrm{m}$ Sestamibi.

The multigland disease has been found to be a significant lesion factor that decreases the diagnostic performance of 4D-CT [14] [16] [18]. As such, we suggest that if one lesion is found, the radiologist should continue to search for other

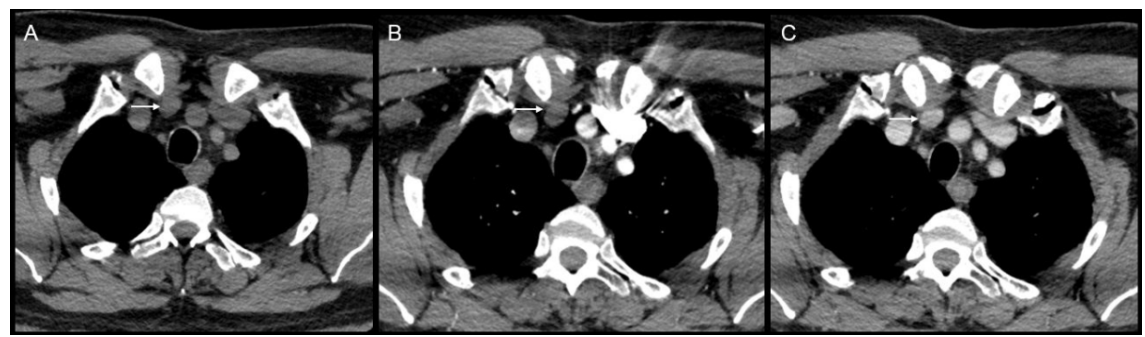

Figure 1. Beam hardening artifact limits adenoma detection on arterial phase. Axial noncontrast (A), arterial (B), and venous (C) CT images showed beam hardening artifact from the IV contrast obscuring the right superior mediastinal adenoma (arrow). The classic intense arterial enhancement of the lesion is not demonstrated due to the artifact. 


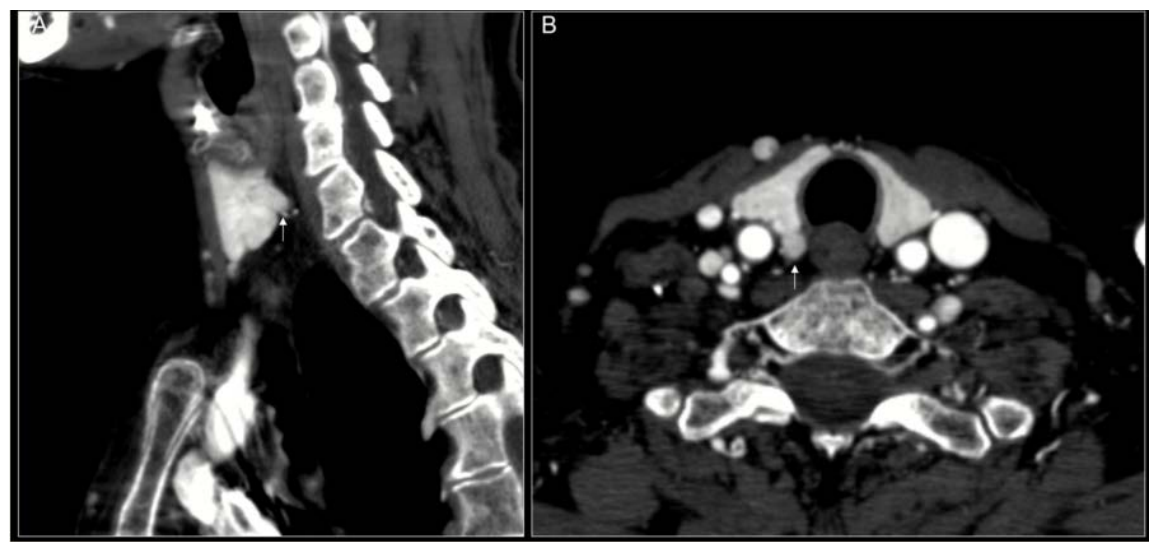

Figure 2. Zuckerkandl tubercle. The normal thyroid gland protruded at the posterolateral aspect (arrow) mimics a parathyroid lesion.

lesions especially in cases of SHPT. Although the small size of the glands is known to be a lesion factor, $4 \mathrm{D}-\mathrm{CT}$ is less affected than Tc-99 $\mathrm{m}$ Sestamibi due to higher intrinsic special resolution [18].

The most common pitfalls (37\%) in our study were lesions that adhered to the posterior capsule of the thyroid (i.e., lack of fat plane between the lesions and the thyroid gland), especially for small lesions that were isodense to the thyroid in all phases. The classic enhancement pattern of parathyroid adenoma/hyperplasia is an avid enhancement on arterial phase followed by contrast wash out on the venous phase. Two lesions were misinterpreted as lymph nodes due to minimal enhancement on the arterial phase and progressive enhancement on the venous phase. Hypodense pedunculated thyroid nodules may simulate parathyroid lesions which accounted for two false positive findings in our cohort.

Zuckerkandl tubercles are protuberances at the posterolateral aspect of a normal thyroid gland that can potentially mimic a parathyroid lesion (Figure 2) [22]. These are isodense to the thyroid gland on all phases. Typically, parathyroid lesions are posterior to the thyroid glands; however, ectopic or unusual locations $(6 \%-16 \%)$ are challenging to detect. These include but are not limited to the following locations: immediately inferior or lateral to the thyroid glands, mediastinum, retropharyngeal space, retroesophageal space, paraesophageal space, carotid space, tracheoesophageal groove, thyroid gland, thymus, and submandibular gland [23].

Our study has some limitations. The radiologists realized that all cases were proven to have positive surgical and pathological findings, and therefore they would try their best to select the location of the lesions rather than providing negative results. Including studies with negative findings would decrease this bias. We did not re-image studies that had artifacts-in so doing, we believe this provided a more realistic situation where radiologists have to make a final decision even when there may be pronounced artifacts. Finally, the number of cases with multigland diseases was relatively small and may not represent the true performance of $4 \mathrm{D}-\mathrm{CT}$ across all practice setting. 


\section{Conclusion}

4D CT parathyroid is a highly accurate imaging method that can facilitate minimally invasive parathyroidectomy in cases of hyperparathyroidism. Improvements in diagnostic performance could be realized through optimization of imaging techniques and familiarity with potential pitfalls.

\section{Conflicts of Interest}

The authors declare no conflicts of interest regarding the publication of this paper.

\section{References}

[1] DeLellis, R.A., Mazzaglia, P. and Mangray, S. (2008) Primary Hyperparathyroidism: A Current Perspective. Archives of Pathology \& Laboratory Medicine, 132, 1251-1262.

[2] Jamal, S.A. and Miller, P.D. 2013) Secondary and Tertiary Hyperparathyroidism. Journal of Clinical Densitometry, 16, 64-68. https://doi.org/10.1016/j.jocd.2012.11.012

[3] Callender, G.G. and Udelsman, R. (2014) Surgery for Primary Hyperparathyroidism. Cancer, 120, 3602-3616. https://doi.org/10.1002/cncr.28891

[4] Chen, J. and Wang, J.D. (2014) Radioguided Parathyroidectomy in Patients with Secondary Hyperparathyroidism Due to Chronic Renal Failure. Nuclear Medicine Communications, 35, 391-397. https://doi.org/10.1097/MNM.0000000000000062

[5] Cappello, Z.J. and Bumpous, J.M. (2013) Is Bilateral Exploration Still the Standard of Care for Primary Hyperparathyroidism? Outcomes of Focused Radio-Guided Parathyroidectomy and Bilateral Explorations. The Laryngoscope, 123, 2587-2588. https://doi.org/10.1002/lary.24084

[6] Sosa, J.A. and Udelsman, R. (2003) New Directions in the Treatment of Patients with Primary Hyperparathyroidism. Current Problems in Surgery, 40, 812-849. https://doi.org/10.1016/S0011-3840(03)00116-3

[7] Lee, S., Ryu, H., Morris, L.F., Lee, S., Ryu, H., Morris, L.F., Grubbs, E.G., Lee, J.E., et al. (2014) Operative Failure in Minimally Invasive Parathyroidectomy Utilizing an Intraoperative Parathyroid Hormone Assay. Annals of Surgical Oncology, 21, 1878-1883. https://doi.org/10.1245/s10434-013-3479-3

[8] Cheung, K., Wang, T.S., Farrokhyar, F., Roman, S.A. and Sosa, J.A. (20212) A Meta-Analysis of Preoperative Localization Techniques for Patients with Primary Hyperparathyroidism. Annals of Surgical Oncology, 19, 577-583. https://doi.org/10.1245/s10434-011-1870-5

[9] Krakauer, M., Wieslander, B., Myschetzky, P.S., Lundstrøm, A., Bacher, T., Sørensen, C.H., et al. (2016) A Prospective Comparative Study of Parathyroid Dual-Phase Scintigraphy, Dual-Isotope Subtraction Scintigraphy, 4D-CT, and Ultrasonography in Primary Hyperparathyroidism. Clinical Nuclear Medicine, 41, 93-100. https://doi.org/10.1097/RLU.0000000000000988

[10] Mallette, L.E., Gomez, L. and Fisher, R.G. (1981) Parathyroid Angiography: A Review of Current Knowledge and Guidelines for Clinical Application. Endocrine Reviews, 2, 124-135. https://doi.org/10.1210/edrv-2-1-124

[11] Hunter, G.J., Schellingerhout, D., Vu, T.H., Perrier, N.D. and Hamberg, L.M. (2012) Accuracy of Four-Dimensional CT for the Localization of Abnormal Parathyroid 
Glands in Patients with Primary Hyperparathyroidism. Radiology, 264, 789-795. https://doi.org/10.1148/radiol.12110852

[12] Takami, H., Ikeda, Y. and Wada, N. (2000) Surgical Management of Primary Hyperparathyroidixsm. Biomedicine \& Pharmacotherapy, 54, 17s-20s. https://doi.org/10.1016/S0753-3322(00)80005-0

[13] Laird, A.M. and Libutti, S.K. (20016) Minimally Invasive Parathyroidectomy versus Bilateral Neck Exploration for Primary Hyperparathyroidism. Surgical Oncologyclinics of North America, 25, 103-118. https://doi.org/10.1016/j.soc.2015.08.012

[14] Chazen, J.L., Gupta, A., Dunning, A. and Phillips, C.D. (2012) Diagnostic Accuracy of 4D-CT for Parathyroid Adenomas and Hyperplasia. American Journal of Neuroradiology, 33, 429-433. https://doi.org/10.3174/ajnr.A2805

[15] Campbell, M.J., Sicuro, P., Blackmore, C.C. and Ryan Jr., J.A. (2015) Two-Phase (Low-Dose) Computed Tomography Is as Effective as 4D-CT for Identifying Enlarged Parathyroid Glands. International Journal of Surgery. 14, 80-84. https://doi.org/10.1016/j.ijsu.2015.01.005

[16] Rodgers, S.E., Hunter, G.J., Hamberg, L.M., Schellingerhout, D., Doherty, D.B. and Ayers, G.D. (2006) Improved Preoperative Planning for Directed Parathyroidectomy with 4-Dimensional Computed Tomography. Surgery, 140, 932-940. https://doi.org/10.1016/j.surg.2006.07.028

[17] Mortenson, M.M., Evans, D.B., Lee, J.E., Hunter, G.J., Shellingerhout, D., Vu, T., et al. (2008) Parathyroid Exploration in the Reoperative Neck: Improved Preoperative Localization with 4D-Computed Tomography. Journal of the American College of Surgeons, 206, 888-895. https://doi.org/10.1016/j.jamcollsurg.2007.12.044

[18] Galvin, L., Oldan, J.D., Bahl, M., Eastwood, J.D., Eastwood, J.D., Sosa, J.A. and Hoang, J.K. (2016) Parathyroid 4D CT and Scintigraphy: What Factors Contribute to Missed Parathyroid Lesions? Otolaryngology_Head and Neck Surgery, 154, 847-853. https://doi.org/10.1177/0194599816630711

[19] Rajapakse, C.S. and Chang, G. (2014) Impact of Body Habitus on Radiologic Interpretations. Academic Radiology, 2, 1-2. https://doi.org/10.1016/j.acra.2013.10.006

[20] Hoang, J.K., Sung, W.K., Bahl, M. and Phillips, C.D. (2014) How to Perform Parathyroid 4D CT: Tips and Traps for Technique and Interpretation. Radiology, 270, 15-24. https://doi.org/10.1148/radiol.13122661

[21] Lubitz, C.C., Hunter, G.J., Hamberg, L.M., Parangi, S., Ruan, D., Gawande, A., et al. (2010) Accuracy of 4-Dimensional Computed Tomography in Poorly Localized Patients with Primary Hyperparathyroidism. Surgery, 148, 1129-1137.

https://doi.org/10.1016/j.surg.2010.09.002

[22] Lee, T.C., Selvarajan, S.K., Curtin, H. and Mukundan, S. (2012) Zuckerkandl Tubercle of the Thyroid: A Common Imaging Finding That May Mimic Pathology. American Journal of Neuroradiology, 33, 1134-1138. https://doi.org/10.3174/ajnr.A2914

[23] Phitayakorn, R. and McHenry, C.R. (2006) Incidence and Location of Ectopic Abnormal Parathyroid Glands. American Journal of Surgery, 191, 418-423.

https://doi.org/10.1016/j.amjsurg.2005.10.049 